This item was submitted to Loughborough's Research Repository by the author.

Items in Figshare are protected by copyright, with all rights reserved, unless otherwise indicated.

\title{
Vector model of vehicle collisions for inferring velocity from loss of kinetic energy with restitution on residual crush surface
}

PLEASE CITE THE PUBLISHED VERSION

http://dx.doi.org/10.1080/13588265.2016.1174439

\section{PUBLISHER}

(C) Taylor \& Francis

\section{VERSION}

AM (Accepted Manuscript)

\section{PUBLISHER STATEMENT}

This work is made available according to the conditions of the Creative Commons Attribution-NonCommercialNoDerivatives 4.0 International (CC BY-NC-ND 4.0) licence. Full details of this licence are available at: https://creativecommons.org/licenses/by-nc-nd/4.0/

\section{LICENCE}

CC BY-NC-ND 4.0

\section{REPOSITORY RECORD}

Lenard, James. 2019. "Vector Model of Vehicle Collisions for Inferring Velocity from Loss of Kinetic Energy with Restitution on Residual Crush Surface". figshare. https://hdl.handle.net/2134/20962. 


\title{
Vector model of vehicle collisions for inferring velocity from loss of kinetic energy with restitution on residual crush surface
}

\author{
James Lenard* \\ Loughborough University, Loughborough, Leics LE11 3TU, UK
}

(March 2016)

\begin{abstract}
In the standard mathematical model that underpins the inference of velocity change from vehicle damage in road accident reconstruction, the point where the colliding bodies engage is taken to lie in the same location as the point of application of the average impact force, usually in the central region of the crush zone or on the residual crush surface. Mathematical and physical reasons suggest the fidelity of the model could be deepened by allowing for a separation of these points, for example by locating the impulse or average force in the central region of the crush zone and defining engagement (common velocity or rebound) relative to the crush surface. Refinement of the theory revealed that the solutions for the change of linear and angular velocity are unaffected. For long-running in-depth research studies, this means that historical calculations of velocity change (delta-V) and related analyses on such topics as injury risk curves, countermeasure effectiveness and accident scenarios are not potentially undermined. Relative and absolute velocity are however affected. This was illustrated using crash test data where adjustments of six and twelve centimetres resulted in changes of up to four percent in road speed.
\end{abstract}

\section{Introduction}

Although much can be inferred about the circumstances of road traffic accidents by considering vehicle rest positions, tyre marks, debris, road surface characteristics and so on, the cost and difficulty of at-scene investigations means that in many cases the best that can be done is to base an accident reconstruction on examination of the crashed vehicles away from the accident scene supported by police reports and eye-witness accounts. Testimony to the enduring importance of damage-based or 'retrospective' methods is provided by their use in large-scale research projects such as those sponsored by NHTSA [16] and the UK Department for Transport [5-7] as well as in litigation, insurance, research and development, and other fields.

Following in a tradition dating back well over fifty years [2, 8, 13, 20-22], colliding vehicles may be represented as two-dimensional rigid bodies whose behaviour is governed by a relatively small number of physical laws and characteristics. In a recent review [18], impact-phase models in common use including the well-known Crash3 algorithm [15] were shown to be essentially equivalent representations of the same model provided for example that the impact plane, which can appear as an explicit parameter $[3,10]$, is understood to be oriented perpendicular to the

*Corresponding author. Email: j.a.lenard@lboro.co.uk 
average impact force.

In every system it is necessary to state how the colliding vehicles move relative to each other. In the simplest case the contacting surfaces fully engage and maintain an equal velocity until separation, a condition referred to as common velocity. In light of empirical evidence that the contacting surfaces of the vehicles may rebound after impact, coefficients of restitution are introduced that allow common velocity as a special case. Common velocity and rebound are defined relative to a single point that represents the whole contacting surface of each vehicle. This point has been placed at different locations along with the point of application of the impulse or average impact force, e.g. in the central region (geometric centroid) of the crush zone $[14,15]$ or on the crush surface $[3,10]$.

At any moment during impact the net force on each vehicle captures the overall effect of loading distributed across the entire contacting surface. The magnitude and direction of this force varies during the course of impact and its point of application also moves (relative to the vehicle's centre of mass) as crush occurs. A detailed study of the location of the impact centre as a function of time was reported in [11] for forty-five car-to-car collision tests based on acceleration, velocity and displacement measurements. It documented significant movements of the impact centre for some crash configurations during the collision phase (50-150 ms at $10 \mathrm{~ms}$ intervals) but found that the impact centre was located near the mid-point of the contacting surface at maximum vehicle deformation. The impulse $\boldsymbol{J}$ on the vehicle as defined in this paper is based in principle on the magnitude, direction and location of the instantaneous net force across the whole collision phase (equations 2.2 and 2.3).

There are a couple of reasons to query whether the restitution point should be located in the middle of the crush zone. Unlike the impact force, which acts from first contact through to maximum crush and is naturally considered to have an average value somewhere in between, the relative velocity of the vehicles at the end of impact depends on their physical configuration and engagement at that time, when the level of crush is high. Secondly there is a mathematical problem. Even in the favourable circumstance where the surfaces of the colliding vehicles fully engage and lock into each other for the duration of impact, although it is known (by hypothesis) that points on the contacting surfaces have the same velocity at any moment of time, this velocity is not linked to the linear and angular velocity of the centre of mass of the vehicles in the required manner while deformation is occurring. The models rely on the relationship $\boldsymbol{v}^{\prime}=\boldsymbol{v}+\boldsymbol{\omega} \times \boldsymbol{r}$ where $\boldsymbol{v}^{\prime}$ is the velocity of the point in question, $\boldsymbol{r}$ is its location relative to the centre of mass and $\boldsymbol{v}$ and $\boldsymbol{\omega}$ are the linear and angular velocity at the centre of mass. For the contacting surfaces of colliding vehicles, this relationship only holds when the deformation pauses or stops, namely at maximum dynamic or residual crush, in either case at or towards the end of the impact phase.

The aim of this paper is to isolate and describe the effect of separating the restitution point from the impulse point. This is implemented by imagining that the impulse point $\mathrm{P}$ lies in the central region of the crush zone and that the restitution point $\mathrm{Q}$ lies on the residual crush surface. The mathematical model employed for this purpose is based on [22] for the assessment of velocity change as extended by [19] to include relative velocity and absolute velocity. This is one of the commonly used, 'essentially equivalent' models surveyed by [18], referred to here as the Smith-Neades or standard model.

In order to focus precisely on the effect of separating restitution from impulse, no other innovation is incorporated into the Smith-Neades model even where variations are described in the literature or otherwise conceivable. This model employs a single coefficient of restitution rather than the two that appear in other formulations and it requires that the impact plane be oriented perpendicular to the direction of impulse. A demonstration of the equivalence of Smith-Neades to other mainstream models $[3,10,15]$ under given conditions is detailed in [18]. Certain other issues are also not addressed here such as how to infer energy dissipation from crush and stiffness (or by other means) or how to locate the point of application of impulse using the crush profile. The established procedures for carrying out these necessary steps of a damage-based accident reconstruction are independent of the focus of this paper - the separation of the impulse point 
$\mathrm{P}$ from the restitution point $\mathrm{Q}$ - and it suffices to assume that the steps have been carried out in a conventional manner.

The mathematics is presented below in a style that maintains the use of full vector terms and vector functions such as the cross product. This suppresses the appearance of trigonometric functions and avoids the need to deal with a multiplicity of terms and equations involving angles and vector components. Hence it is very amenable to exploring displacement of the restitution point and other theoretical refinements. It has been said of the use of vector algebra in accident reconstruction that 'the reward for its abstraction lies in the refreshing clarity and brevity of the derivations' [9]. The next section begins with a review of the Smith-Neades model followed by a demonstration of how it may be adapted to deal with separation of the restitution point from the point of application of the average impact force. It turns out that when the standard model is set out in this way, it can be seen to apply virtually lock, stock and barrel to the refined model.

\section{Materials and Method}

At the core of a damage-based reconstruction, the change of velocity during impact (delta-V) is inferred from vehicle damage using just a few items of information: mass $m$, moment of inertia $I$, direction of impulse $\hat{\boldsymbol{J}}$ (principal direction of force) and location of impulse $\boldsymbol{R}$ for each vehicle; in addition it is necessary to know the total loss of kinetic energy $\Delta K$ and the coefficient of restitution $e$ (see figure 1). Models that omit reference to restitution by building in common velocity of the contacting regions can be interpreted as implicitly setting $e$ to zero. Residual damage including depth of crush is often used to identify the direction and location of impulse and, in conjunction with stiffness parameters, to estimate kinetic energy loss. In this sense crush is a very important item that lies behind at least three of the parameters listed but in the interests of brevity it is not drawn directly into the discussion below.

The standard model of vehicle collisions can be derived from five equations, three of which are formulas of classical mechanics for a single rigid body (equations 2.1, 2.2 and 2.3) and the two of which state a relationship between the colliding bodies (equations 2.4 and 2.5). A full explanation of equations 2.1 to 2.4 can be found in standard textbooks [1].

The change of kinetic energy for a single body is the difference between its initial and final kinetic energy:

$$
\Delta K=\left(\frac{1}{2} m \boldsymbol{v}_{f}^{2}+\frac{1}{2} I \boldsymbol{\omega}_{f}^{2}\right)-\left(\frac{1}{2} m \boldsymbol{v}_{i}^{2}+\frac{1}{2} I \boldsymbol{\omega}_{i}^{2}\right)
$$

The impulse $\boldsymbol{J}$ on a body is defined as the integral of the force $\boldsymbol{F}$ at each moment of time over the duration of impact $t_{i}$ to $t_{f}$; this equals the change of momentum $m \Delta \boldsymbol{v}$ :

$$
\boldsymbol{J}=\int_{t_{i}}^{t_{f}} \boldsymbol{F} \mathrm{d} t=m \Delta \boldsymbol{v}
$$

Similarly the integral of the torque $\boldsymbol{\tau}=\boldsymbol{r} \times \boldsymbol{F}$ on a body over the duration of impact equals the change of angular momentum $I \Delta \boldsymbol{\omega}$ and $\boldsymbol{R}$ is defined as a position relative to the centre of mass at which the impulse $\boldsymbol{J}$ has the same effect:

$$
\boldsymbol{R} \times \boldsymbol{J}=\int_{t_{i}}^{t_{f}}(\boldsymbol{r} \times \boldsymbol{F}) \mathrm{d} t=I \Delta \boldsymbol{\omega}
$$

It is required that the two colliding bodies constitute an isolated system, i.e. they are not subject to net forces from other bodies and therefore that the impulses on the vehicles are equal 

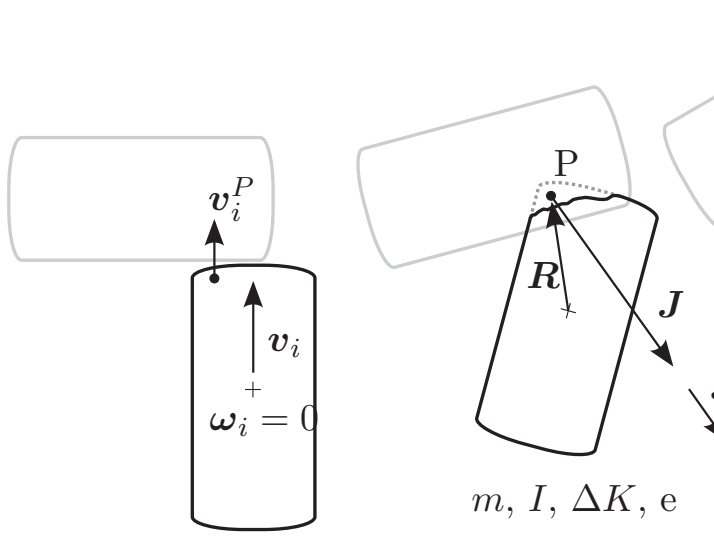

$m, I, \Delta K, \mathrm{e}$
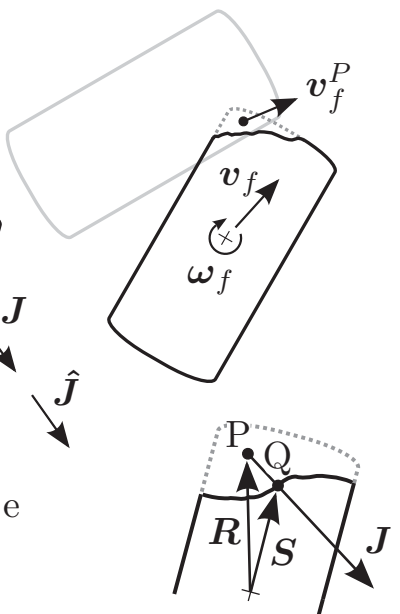

Figure 1. Schematic of vehicle collision showing pre-impact velocities (left), impact phase parameters (centre), post-impact velocities (right) and separation of restitution point (lower right) for one of the vehicles:

$\begin{array}{llll}\boldsymbol{v}_{i} & \text { initial velocity } & m & \text { mass } \\ \hat{\boldsymbol{v}}_{i} & \text { initial velocity (unit vector) } & I & \text { moment of inertia } \\ \boldsymbol{v}_{i}^{P} & \text { initial velocity at P } & \Delta K & \text { change of kinetic energy } \\ \boldsymbol{\omega}_{i} & \text { initial angular velocity } & \mathrm{P} & \text { impulse point } \\ \boldsymbol{v}_{f} & \text { final velocity } & \boldsymbol{R} & \text { location of impulse } \\ \boldsymbol{v}_{f}^{P} & \text { final velocity at } \mathrm{P} & \boldsymbol{J} & \text { impulse } \\ \boldsymbol{\omega}_{f} & \text { final angular velocity } & \hat{\boldsymbol{J}} & \text { impulse (unit vector) } \\ \Delta \boldsymbol{v} & \text { change of velocity } & \mathrm{Q} & \text { restitution point } \\ e & \text { coefficient of restitution } & \boldsymbol{S} & \text { location of restitution }\end{array}$

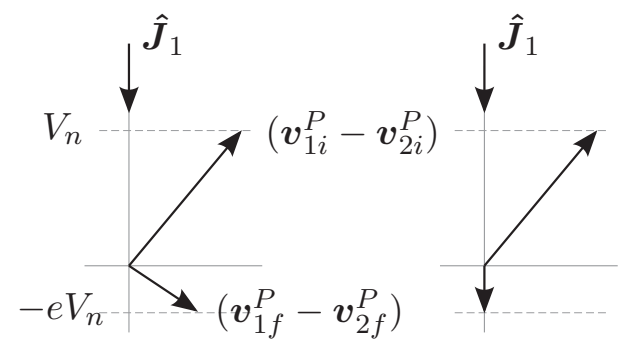

Figure 2. (left) Coefficient of restitution $e$ specifies that the component of relative velocity parallel to impulse $\hat{\boldsymbol{J}}$ changes from $V_{n}$ at the contact point before impact to $-e V_{n}$ after impact; (right) with added condition that there is no component perpendicular to impulse after impact.

in magnitude and opposite in direction:

$$
\boldsymbol{J}_{1}+\boldsymbol{J}_{2}=0
$$

This condition is generally satisfied if the frictional forces between the tyres and the road surface are negligible compared to the direct collision forces.

A second condition on the interaction of the colliding bodies is set by a coefficient of restitution $e$ that relates the relative velocity of the vehicles at the impulse point $\mathrm{P}$ before and after impact. A common definition [22] specifies that the component of this relative velocity in the direction of impulse is opposite in direction and lower in magnitude by the factor $e$ after impact:

$$
\left(\boldsymbol{v}_{1 f}^{P}-\boldsymbol{v}_{2 f}^{P}\right) \cdot \hat{\boldsymbol{J}}_{1}=-e\left(\boldsymbol{v}_{1 i}^{P}-\boldsymbol{v}_{2 i}^{P}\right) \cdot \hat{\boldsymbol{J}}_{1}
$$

where $0 \leq e \leq 1$ (figure 2, left). This definition places no constraint on the component of relative velocity perpendicular to the direction of impulse before or after impact. The special case of common velocity at the impulse point $\mathrm{P}$ after impact, $\boldsymbol{v}_{1 f}^{P}=\boldsymbol{v}_{2 f}^{P}$, is expressed by $e=0$. 


\section{Results}

\subsection{Standard model with restitution at impulse point}

The derivations in this section provide solutions for velocity change, relative velocity and absolute velocity based on the conventional assumption that restitution or common velocity applies at the impulse point $\mathrm{P}$.

\subsubsection{Change of velocity}

From equation 2.1, the change of kinetic energy for a single body is

$$
\begin{aligned}
\Delta K & =\frac{1}{2} m\left(\boldsymbol{v}_{f}^{2}-\boldsymbol{v}_{i}^{2}\right)+\frac{1}{2} I\left(\boldsymbol{\omega}_{f}^{2}-\boldsymbol{\omega}_{i}^{2}\right) \\
& =\frac{1}{2} m\left(\boldsymbol{v}_{f}-\boldsymbol{v}_{i}\right) \cdot\left(\boldsymbol{v}_{f}+\boldsymbol{v}_{i}\right)+\frac{1}{2} I\left(\boldsymbol{\omega}_{f}-\boldsymbol{\omega}_{i}\right) \cdot\left(\boldsymbol{\omega}_{f}+\boldsymbol{\omega}_{i}\right)
\end{aligned}
$$

Eliminating $\boldsymbol{v}_{i}$ using $\boldsymbol{v}_{i}=\boldsymbol{v}_{f}-\Delta \boldsymbol{v}$

$$
\begin{aligned}
\Delta K & =\frac{1}{2}\left(m \Delta \boldsymbol{v} \cdot\left(2 \boldsymbol{v}_{f}-\Delta \boldsymbol{v}\right)+I \Delta \boldsymbol{\omega} \cdot\left(2 \boldsymbol{\omega}_{f}-\Delta \boldsymbol{\omega}\right)\right) \\
& =m \Delta \boldsymbol{v} \cdot \boldsymbol{v}_{f}+I \Delta \boldsymbol{\omega} \cdot \boldsymbol{\omega}_{f}-\frac{1}{2}\left(\frac{1}{m}(m \Delta \boldsymbol{v})^{2}+\frac{1}{I}(I \Delta \boldsymbol{\omega})^{2}\right)
\end{aligned}
$$

Substituting in $\boldsymbol{J}=m \Delta \boldsymbol{v}$ (equation 2.2) and $\boldsymbol{R} \times \boldsymbol{J}=I \Delta \boldsymbol{\omega}$ (equation 2.3),

$$
\begin{aligned}
\Delta K & =\boldsymbol{J} \cdot \boldsymbol{v}_{f}+(\boldsymbol{R} \times \boldsymbol{J}) \cdot \boldsymbol{\omega}_{f}-\frac{1}{2}\left(\frac{1}{m} \boldsymbol{J}^{2}+\frac{1}{I}(\boldsymbol{R} \times \boldsymbol{J})^{2}\right) \\
& =\boldsymbol{v}_{f} \cdot \boldsymbol{J}+\left(\boldsymbol{\omega}_{f} \times \boldsymbol{R}\right) \cdot \boldsymbol{J}-\frac{1}{2}\left(\frac{1}{m} \boldsymbol{J}^{2}+\frac{1}{I}(\boldsymbol{R} \times \boldsymbol{J})^{2}\right)
\end{aligned}
$$

Inserting $\boldsymbol{v}_{f}^{P}=\boldsymbol{v}_{f}+\boldsymbol{\omega}_{f} \times \boldsymbol{R}$ and separating the magnitude of impulse $\|\boldsymbol{J}\|$ implicit in $\boldsymbol{J}^{2}$ from the unit vector $\hat{\boldsymbol{J}}$,

$$
\begin{aligned}
\Delta K & =\boldsymbol{v}_{f}^{P} \cdot \boldsymbol{J}-\frac{1}{2}\left(\frac{1}{m}+\frac{1}{I}(\boldsymbol{R} \times \hat{\boldsymbol{J}})^{2}\right) J^{2} \\
& =\boldsymbol{v}_{f}^{P} \cdot \boldsymbol{J}-a J^{2}
\end{aligned}
$$

where

$$
a=\frac{1}{2}\left(\frac{1}{m}+\frac{1}{I}(\boldsymbol{R} \times \hat{\boldsymbol{J}})^{2}\right)
$$

There is a free choice to emphasize either the vector or scalar nature of $\boldsymbol{J} \cdot \boldsymbol{J}=\|\boldsymbol{J}\|^{2}$, written in abbreviated form as $\boldsymbol{J}^{2}=J^{2}$. The term $a$, like $E$ below, is introduced to shorten the following equations and show their structure more clearly. Its value is determined by quantities assumed to be known in damage-based accident reconstruction.

If $\boldsymbol{v}_{f}$ is eliminated above instead of $\boldsymbol{v}_{i}$,

$$
\Delta K=\frac{1}{2}\left(m \Delta \boldsymbol{v} \cdot\left(2 \boldsymbol{v}_{i}+\Delta \boldsymbol{v}\right)+I \Delta \boldsymbol{\omega} \cdot\left(2 \boldsymbol{\omega}_{i}+\Delta \boldsymbol{\omega}\right)\right)
$$

and parallel reasoning produces

$$
\Delta K=\boldsymbol{v}_{i}^{P} \cdot \boldsymbol{J}+a J^{2}
$$


Drawing on equations 3.1 and 3.2 in turn, and moving on from a single body to the total change of kinetic energy for two bodies,

$$
\begin{aligned}
-E=\Delta K_{1}+\Delta K_{2} & =\left(\boldsymbol{v}_{1 f}^{P} \cdot \boldsymbol{J}_{1}-a_{1} J_{1}^{2}\right)+\left(\boldsymbol{v}_{2 f}^{P} \cdot \boldsymbol{J}_{2}-a_{2} J_{2}^{2}\right) \\
& =\left(\boldsymbol{v}_{1 i}^{P} \cdot \boldsymbol{J}_{1}+a_{1} J_{1}^{2}\right)+\left(\boldsymbol{v}_{2 i}^{P} \cdot \boldsymbol{J}_{2}+a_{2} J_{2}^{2}\right)
\end{aligned}
$$

where $E$ expresses loss of total kinetic energy in a positive sense. For colliding bodies in an isolated system (equation 2.4), $\boldsymbol{J}_{2}=-\boldsymbol{J}_{1}$ and $J_{2}^{2}=J_{1}^{2}$, allowing the elimination of $\boldsymbol{J}_{2}$ :

$$
\begin{aligned}
-E & =\left(\boldsymbol{v}_{1 f}^{P}-\boldsymbol{v}_{2 f}^{P}\right) \cdot \boldsymbol{J}_{1}-\left(a_{1}+a_{2}\right) J_{1}^{2} \\
& =\left(\boldsymbol{v}_{1 i}^{P}-\boldsymbol{v}_{2 i}^{P}\right) \cdot \boldsymbol{J}_{1}+\left(a_{1}+a_{2}\right) J_{1}^{2}
\end{aligned}
$$

Equations 3.3 and 3.4 can be combined to exploit the relationship between $\left(\boldsymbol{v}_{1 i}^{P}-\boldsymbol{v}_{2 i}^{P}\right)$ and $\left(\boldsymbol{v}_{1 f}^{P}-\boldsymbol{v}_{2 f}^{P}\right)$ in the condition for rebound (equation 2.5) and thereby eliminate these terms, namely by multiplying equation 3.4 by $e$ and adding to equation 3.3

$$
\begin{aligned}
-(1+e) E & =\left(\left(\boldsymbol{v}_{1 f}^{P}-\boldsymbol{v}_{2 f}^{P}\right)+e\left(\boldsymbol{v}_{1 i}^{P}-\boldsymbol{v}_{2 i}^{P}\right)\right) \cdot \boldsymbol{J}_{1}-(1-e)\left(a_{1}+a_{2}\right) J_{1}^{2} \\
& =\mathbf{0} \cdot \boldsymbol{J}_{1}-(1-e)\left(a_{1}+a_{2}\right) J_{1}^{2} \\
\frac{(1+e)}{(1-e)} E & =\left(a_{1}+a_{2}\right) J_{1}^{2}
\end{aligned}
$$

Substituting back in for the placeholders $a_{1}$ and $a_{2}$,

$$
\frac{(1+e)}{(1-e)} E=\frac{1}{2}\left[\frac{1}{m_{1}}+\frac{1}{I_{1}}\left(\boldsymbol{R}_{1} \times \hat{\boldsymbol{J}}_{1}\right)^{2}+\frac{1}{m_{2}}+\frac{1}{I_{2}}\left(\boldsymbol{R}_{2} \times \hat{\boldsymbol{J}}_{2}\right)^{2}\right] J_{1}^{2}
$$

This provides a solution for the unknown magnitude of impulse $J_{1} \equiv\left\|\boldsymbol{J}_{1}\right\|$ because the direction of impulse $\hat{\boldsymbol{J}}$ and other terms in the equation are known.

The change of velocity terms for each body $(\Delta \boldsymbol{v}, \Delta \omega)$ follow directly from equations 2.2 and 2.3 using $\boldsymbol{J}_{1}=\left\|\boldsymbol{J}_{1}\right\| \hat{\boldsymbol{J}}_{1}$ and $\boldsymbol{J}_{2}=-\boldsymbol{J}_{1}$.

\subsubsection{Relative velocity}

Further information is required to solve for the relative velocity between the colliding vehicles. The standard model [19] considers the case where there is no relative movement between the vehicles at point $\mathrm{P}$ perpendicular to the direction of impulse after impact (figure 2, right) which may be expressed as

$$
\left(\boldsymbol{v}_{1 f}^{P}-\boldsymbol{v}_{2 f}^{P}\right)=\left(\boldsymbol{v}_{1 f}^{P}-\boldsymbol{v}_{2 f}^{P}\right) \cdot \hat{\boldsymbol{J}}_{1} \hat{\boldsymbol{J}}_{1}
$$

The definition of restitution (equation 2.5) can be restated as

$$
\left(\boldsymbol{v}_{1 f}^{P}-\boldsymbol{v}_{2 f}^{P}\right) \cdot \hat{\boldsymbol{J}}_{1}=\frac{e}{1+e}\left(\Delta \boldsymbol{v}_{2}^{P}-\Delta \boldsymbol{v}_{2}^{P}\right) \cdot \hat{\boldsymbol{J}}_{1}
$$

by eliminating each $\boldsymbol{v}_{i}^{P}$ term using $\boldsymbol{v}_{i}^{P}=\boldsymbol{v}_{f}^{P}-\Delta \boldsymbol{v}^{P}$. Multiplying both sides by $\hat{\boldsymbol{J}}_{1}$ and simplifying the left hand side using equation 3.6 provides a solution for the relative velocity of the vehicles at $\mathrm{P}$ after impact:

$$
\left(\boldsymbol{v}_{1 f}^{P}-\boldsymbol{v}_{2 f}^{P}\right)=\frac{e}{(1+e)}\left(\Delta \boldsymbol{v}_{1}^{P}-\Delta \boldsymbol{v}_{2}^{P}\right) \cdot \hat{\boldsymbol{J}}_{1} \hat{\boldsymbol{J}}_{1}
$$




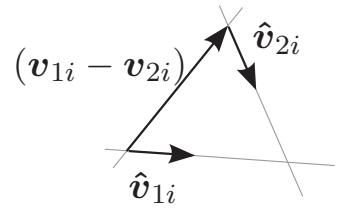

Figure 3. Vector triangulation on the relative velocity before impact $\left(\boldsymbol{v}_{1 i}-\boldsymbol{v}_{2 i}\right)$ to solve for initial road speed using pre-impact trajectories $\hat{\boldsymbol{v}}_{1 i}$ and $\hat{\boldsymbol{v}}_{2 i}$.

since the change of velocity terms $\Delta \boldsymbol{v}^{P}$ are known. The relative velocity at $\mathrm{P}$ before impact is given by the identity

$$
\left(\boldsymbol{v}_{1 i}^{P}-\boldsymbol{v}_{2 i}^{P}\right)=\left(\boldsymbol{v}_{1 f}^{P}-\boldsymbol{v}_{2 f}^{P}\right)-\left(\Delta \boldsymbol{v}_{1}^{P}-\Delta \boldsymbol{v}_{2}^{P}\right)
$$

If it can be further established that the vehicles were not rotating before impact, i.e. that $\boldsymbol{\omega}_{1 i}=0$ and $\boldsymbol{\omega}_{2 i}=0$, the relative velocity between the vehicles at the centre of mass before impact is determined by

$$
\left(\boldsymbol{v}_{1 i}-\boldsymbol{v}_{2 i}\right)=\left(\boldsymbol{v}_{1 i}^{P}-\boldsymbol{v}_{2 i}^{P}\right)
$$

since without rotation every point on each vehicle has the same velocity; after impact the relative velocity is

$$
\left(\boldsymbol{v}_{1 f}-\boldsymbol{v}_{2 f}\right)=\left(\boldsymbol{v}_{1 i}-\boldsymbol{v}_{2 i}\right)+\left(\Delta \boldsymbol{v}_{1}-\Delta \boldsymbol{v}_{2}\right)
$$

\subsubsection{Absolute velocity}

If the pre-impact directions of travel $\hat{\boldsymbol{v}}_{1 i}$ and $\hat{\boldsymbol{v}}_{2 i}$ are known, vector triangulation on the relative velocity $\left(\boldsymbol{v}_{1 i}-\boldsymbol{v}_{2 i}\right)$ provides the initial road speeds provided that the trajectories are not parallel (figure 3). It may be convenient to program the solution for the magnitude of the pre-impact velocity vectors using the standard linear algebra form $\mathbf{A} \boldsymbol{x}=\boldsymbol{b}$ :

$$
\left[\hat{\boldsymbol{v}}_{1 i} \hat{\boldsymbol{v}}_{2 i}\right]\left[\begin{array}{c}
\left\|\boldsymbol{v}_{1 i}\right\| \\
-\left\|\boldsymbol{v}_{2 i}\right\|
\end{array}\right]=\left[\left(\boldsymbol{v}_{1 i}-\boldsymbol{v}_{2 i}\right)\right]
$$

Once the pre-impact velocities are determined, the post-impact velocity of each vehicle follows from $\boldsymbol{v}_{f}=\boldsymbol{v}_{i}+\Delta \boldsymbol{v}$.

\section{$3.2 \quad$ Refined model with restitution on crush surface}

The effect of separating restitution from impulse is investigated in this section by assuming that that restitution or common velocity applies at the point $\mathrm{Q}$ where the residual crush surface is intersected by the line of action of impulse (figure 1). The location of Q is defined by $\boldsymbol{S}$ where $\boldsymbol{S}=\boldsymbol{R}+c \hat{\boldsymbol{J}}$ for a value $c$ that is determined in each case by the crush profile and impulse $\hat{\boldsymbol{J}}$.

The condition for restitution (equation 2.5) is adapted accordingly,

$$
\left(\boldsymbol{v}_{1 f}^{Q}-\boldsymbol{v}_{2 f}^{Q}\right) \cdot \hat{\boldsymbol{J}}_{1}=-e\left(\boldsymbol{v}_{1 i}^{Q}-\boldsymbol{v}_{2 i}^{Q}\right) \cdot \hat{\boldsymbol{J}}_{1}
$$

and equations 2.1 to 2.4, which constitute the rest of the mathematical model, apply as for the standard model. 


\subsubsection{Change of velocity}

The key to obtaining a solution for change of velocity with restitution at $\mathrm{Q}$ is to recognise that $\boldsymbol{v}^{Q} \cdot \hat{\boldsymbol{J}}=\boldsymbol{v}^{P} \cdot \hat{\boldsymbol{J}}$ for each vehicle at any given time. To take a specific case,

$$
\begin{aligned}
\boldsymbol{v}_{1 i}^{Q} \cdot \hat{\boldsymbol{J}}_{\mathbf{1}} & =\left(\boldsymbol{v}_{1 i}+\boldsymbol{\omega}_{1 i} \times \boldsymbol{S}_{1}\right) \cdot \hat{\boldsymbol{J}}_{1} \\
& =\left(\boldsymbol{v}_{1 i}+\boldsymbol{\omega}_{1 i} \times\left(\boldsymbol{R}_{1}+c \hat{\boldsymbol{J}}_{1}\right) \cdot \hat{\boldsymbol{J}}_{1}\right. \\
& =\left(\boldsymbol{v}_{1 i}+\boldsymbol{\omega}_{1 i} \times \boldsymbol{R}_{1}\right) \cdot \hat{\boldsymbol{J}}_{1}+\left(\boldsymbol{\omega}_{1 i} \times c \hat{\boldsymbol{J}}_{1}\right) \cdot \hat{\boldsymbol{J}}_{1} \\
& =\boldsymbol{v}_{1 i}^{P} \cdot \hat{\boldsymbol{J}}_{\mathbf{1}}+c\left(\hat{\boldsymbol{J}}_{\mathbf{1}} \times \hat{\boldsymbol{J}}_{1}\right) \cdot \boldsymbol{\omega}_{1 i} \\
& =\boldsymbol{v}_{1 i}^{P} \cdot \hat{\boldsymbol{J}}_{\mathbf{1}}
\end{aligned}
$$

since the cross product of $\hat{\boldsymbol{J}}_{1}$ with itself is necessarily zero. The same applies to vehicle 2 and time $t_{f}$. This allows each $\boldsymbol{v}^{Q} \cdot \hat{\boldsymbol{J}}$ term in equation 3.11 to be substituted by its $\boldsymbol{v}^{P} \cdot \hat{\boldsymbol{J}}$ counterpart, the result being

$$
\left(\boldsymbol{v}_{1 f}^{P}-\boldsymbol{v}_{2 f}^{P}\right) \cdot \hat{\boldsymbol{J}}_{1}=-e\left(\boldsymbol{v}_{1 i}^{P}-\boldsymbol{v}_{2 i}^{P}\right) \cdot \hat{\boldsymbol{J}}_{1}
$$

which is equation 2.5, restitution at impulse point $\mathrm{P}$. In other words, if restitution holds at a point Q lying on the line of action of impulse, it also holds at the impulse point $\mathrm{P}$ itself.

The upshot is that the solution for change of velocity presented for the standard model in section 3.1.1 applies in its entirety to the refined model with restitution at point Q. The first four premises of the model (equations 2.1 to 2.4) are shared and the fifth premise of the standard model, restitution at $\mathrm{P}$ (equation 2.5), is implied by restitution at $\mathrm{Q}$ (equation 3.11).

\subsubsection{Relative velocity}

The solution for relative velocity on the standard model begins with the supposition that there is no relative movement between the vehicles perpendicular to the direction of impulse after impact (equation 3.6). With restitution on the residual crush surface at Q, this becomes

$$
\left(\boldsymbol{v}_{1 f}^{Q}-\boldsymbol{v}_{2 f}^{Q}\right)=\left(\boldsymbol{v}_{1 f}^{Q}-\boldsymbol{v}_{2 f}^{Q}\right) \cdot \hat{\boldsymbol{J}}_{1} \hat{\boldsymbol{J}}_{1}
$$

From this starting point, the solution for relative velocity provided for the standard model in section 3.1.2 above can be mimicked by replacing each $\boldsymbol{v}^{P}$ term with its $\boldsymbol{v}^{Q}$ counterpart because nothing there depends on $\mathrm{P}$ being the point of application of impulse. This leads to the solutions for relative velocity at $\mathrm{Q}$ corresponding to equations 3.7 and 3.8:

$$
\left(\boldsymbol{v}_{1 f}^{Q}-\boldsymbol{v}_{2 f}^{Q}\right)=\frac{e}{(1+e)}\left(\Delta \boldsymbol{v}_{1}^{Q}-\Delta \boldsymbol{v}_{2}^{Q}\right) \cdot \hat{\boldsymbol{J}}_{1} \hat{\boldsymbol{J}}_{1}
$$

and

$$
\left(\boldsymbol{v}_{1 i}^{Q}-\boldsymbol{v}_{2 i}^{Q}\right)=\left(\boldsymbol{v}_{1 f}^{Q}-\boldsymbol{v}_{2 f}^{Q}\right)-\left(\Delta \boldsymbol{v}_{1}^{Q}-\Delta \boldsymbol{v}_{2}^{Q}\right)
$$

If the vehicles are not rotating before impact, the solutions for relative velocity at the centre of mass are

$$
\left(\boldsymbol{v}_{1 i}-\boldsymbol{v}_{2 i}\right)=\left(\boldsymbol{v}_{1 i}^{Q}-\boldsymbol{v}_{2 i}^{Q}\right)
$$


Table 1. Illustrative case with sample data for damage-based reconstruction and vehicle configuration at impact.

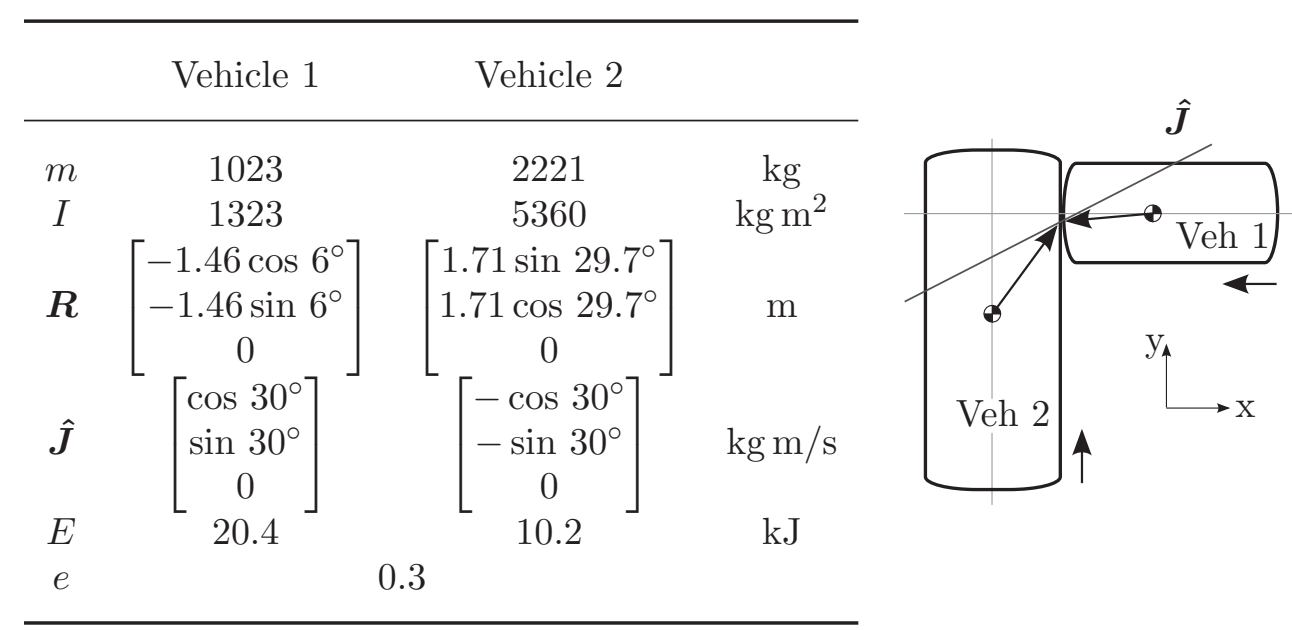

and

$$
\left(\boldsymbol{v}_{1 f}-\boldsymbol{v}_{2 f}\right)=\left(\boldsymbol{v}_{1 i}-\boldsymbol{v}_{2 i}\right)+\left(\Delta \boldsymbol{v}_{1}-\Delta \boldsymbol{v}_{2}\right)
$$

corresponding to equations 3.9 and 3.10 .

It can be shown that $\left(\boldsymbol{v}_{1 f}^{Q}-\boldsymbol{v}_{2 f}^{Q}\right)=\left(\boldsymbol{v}_{1 f}^{P}-\boldsymbol{v}_{2 f}^{P}\right)$ using the equivalence of $\boldsymbol{v}^{Q} \cdot \hat{\boldsymbol{J}}$ to $\boldsymbol{v}^{P} \cdot \hat{\boldsymbol{J}}$ noted above, however in general the other relative velocities will differ depending on whether $\mathrm{P}$ or $\mathrm{Q}$ is taken as the point of restitution.

\subsubsection{Absolute velocity}

The use of vector triangulation to solve for absolute velocity (figure 3) makes no reference to the points $\mathrm{P}$ or $\mathrm{Q}$ and so the technique outlined for the standard model in section 3.1.3 applies without alteration. In general absolute velocity (road speed) will differ depending on the location of the restitution point.

\subsection{Illustrative case}

The effect of separating the restitution point $\mathrm{Q}$ from the impulse point $\mathrm{P}$ is illustrated in this section using sample data from a well known series of crash tests (RICSAC 9). The configuration of the vehicles at impact and the information required to simulate the relevant part of a damagebased reconstruction is drawn from the literature $[4,17,18]$ as presented in table 1 . The impulse location $\boldsymbol{R}$ and direction $\hat{\boldsymbol{J}}$ are left as trigonometic expressions so that the vectors can be easily visualised and linked to the reference sources. Energy dissipation values appear separately for each vehicle although only the total loss of kinetic energy is used in the calculation. The restitution value of $e=0.3$ was found by [18] to provide best fit to the experimental test readings.

A selection of results obtained by running the reconstruction data through the equations for the standard model is shown in the upper row of table 2 . The magnitude of each velocity (speed) is shown below each vector. The results match the values cited in [18] to $\pm 0.01 \mathrm{~m} / \mathrm{s}$, including change of linear and angular velocity $(\|\Delta \boldsymbol{v}\|, \Delta \boldsymbol{\omega})$, pre-impact velocity $\left(\boldsymbol{v}_{i}\right)$ and post-impact velocity $\left(\boldsymbol{v}_{f}\right)$; from this it follows that the relative velocities also match.

The lower row of table 2 shows a set of revised results obtained on the assumption that the restitution point lies on the residual crush surface. Based on the crush profiles for vehicles 1 and 2 with mean values of $20.3 \mathrm{~cm}$ and $10.5 \mathrm{~cm}$ respectively [12, 17], the point of restitution on the residual crush surface was determined for each vehicle relative to the impulse point as 
Table 2. Velocity results of the sample damage-based reconstruction with restitution located at the impulse point or on the residual crush surface $(\mathrm{m} / \mathrm{s})$.

\begin{tabular}{|c|c|c|c|c|c|c|}
\hline \multicolumn{4}{|c|}{ Vehicle 1} & \multicolumn{3}{|c|}{ Vehicle 2} \\
\hline$\Delta \boldsymbol{v}_{1}$ & $\left(\boldsymbol{v}_{1 i}-\boldsymbol{v}_{2 i}\right)$ & $\boldsymbol{v}_{1 i}$ & $\boldsymbol{v}_{1 f}$ & $\Delta \boldsymbol{v}_{2}$ & $\boldsymbol{v}_{2 i}$ & $\boldsymbol{v}_{2 f}$ \\
\hline \multicolumn{7}{|c|}{ Restitution at impulse point } \\
\hline$[6.66]$ & {$[-8.18]$} & {$[-8.18]$} & {$[-1.52]$} & {$[-3.07]$} & {$[0]$} & $\lceil-3.07$ \\
\hline 3.85 & -8.04 & 0 & 3.85 & -1.77 & 8.04 & 6.26 \\
\hline 0 & 0 & 0 & 0 & 0 & 0 & 0 \\
\hline 7.70 & $11.47^{\lrcorner}$ & 8.18 & ${ }^{L} 4.14$ & 3.54 & 8.04 & $6.98^{-}$ \\
\hline \multicolumn{4}{|c|}{$\Delta \omega_{1}=-203 \mathrm{deg} / \mathrm{s}$} & \multicolumn{3}{|c|}{$\Delta \omega_{2}=72 \mathrm{deg} / \mathrm{s}$} \\
\hline \multicolumn{7}{|c|}{ Restitution on residual crush surface } \\
\hline$[6.66]$ & {$[-8.36]$} & {$[-8.36]$} & {$[-1.69]$} & {$[-3.07]$} & 0 & {$[-3.07$} \\
\hline 3.85 & -7.73 & 0 & 3.85 & -1.77 & 7.73 & 5.96 \\
\hline 0 & 0 & 0 & 0 & 0 & 0 & 0 \\
\hline 7.70 & 11.39 & 8.36 & 4.20 & 3.54 & 7.73 & 6.71 \\
\hline \multicolumn{4}{|c|}{$\Delta \omega_{1}=-203 \mathrm{deg} / \mathrm{s}$} & \multicolumn{3}{|c|}{$\Delta \omega_{2}=72 \mathrm{deg} / \mathrm{s}$} \\
\hline
\end{tabular}

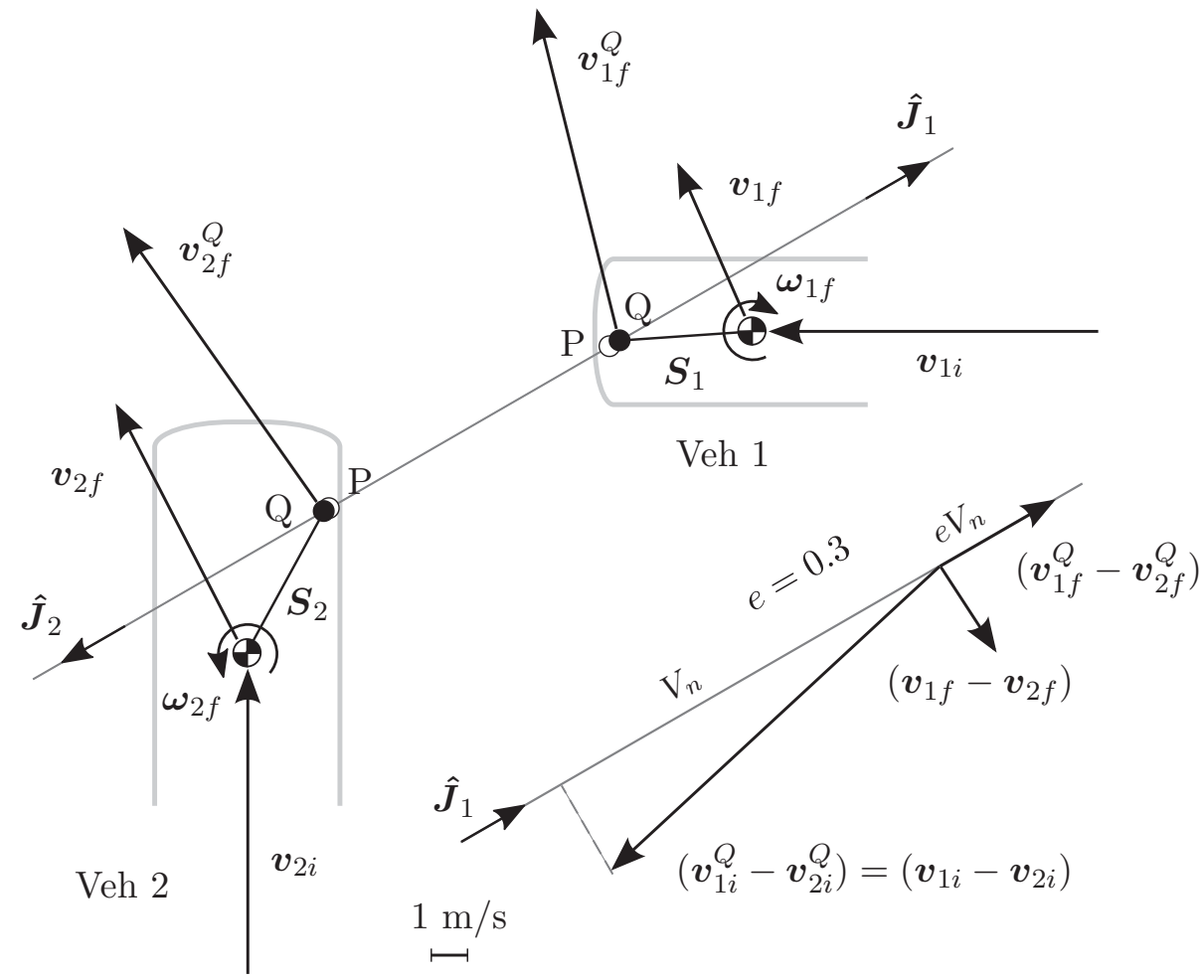

Figure 4. True-to-scale diagram of the results of the sample damage-based reconstruction with restitution located on the residual crush surface.

$\boldsymbol{S}=\boldsymbol{R}+c \hat{\boldsymbol{J}}$ with distance $c$ along the thirty-degree line of action at $12 \mathrm{~cm}$ and $6 \mathrm{~cm}$ for vehicles 1 and 2 respectively $\left(\boldsymbol{S}_{1}=[-1.35 ;-0.09 ; 0.00], \boldsymbol{S}_{2}=[0.79 ; 1.45 ; 0.00]\right)$. The change of linear and angular velocity with restitution at $\mathrm{Q}$ is necessarily unchanged. Looking at the magnitude of the other velocities in this example, the closing speed is slightly reduced $(11.47$ to $11.39 \mathrm{~m} / \mathrm{s})$ while the road speeds of vehicle $1(8.18$ and $4.14 \mathrm{~m} / \mathrm{s})$ rise by $1-2 \%$ and the road speeds of vehicle 2 $(8.04$ and $6.98 \mathrm{~m} / \mathrm{s})$ drop by about $4 \%$.

Further insight into the workings of the model as exemplified by this illustrative case can be 
gained from figure 4 which shows a selection of velocity and position vectors drawn accurately to scale. The restitution point $Q$ is displaced from the impulse point $P$ in the direction of the impulse $\boldsymbol{J}$. In this collision the post-impact velocity $\boldsymbol{v}_{f}^{Q}$ of each vehicle at $Q$ was increased in magnitude and altered slightly in direction compared to the velocity $\boldsymbol{v}_{f}$ at the centre of mass by the action of the post-impact rotation $\boldsymbol{\omega}_{f}$. On the lower right-hand side of figure 4 it can be seen that the difference $\left(\boldsymbol{v}_{1 f}^{Q}-\boldsymbol{v}_{2 f}^{Q}\right)$ between the post-impact velocities of the vehicles at $Q$ is parallel to the direction of impulse $\hat{\boldsymbol{J}}_{1}$ and is related by the factor $e$, the coefficient of restitution, to the difference $\left(\boldsymbol{v}_{1 i}^{Q}-\boldsymbol{v}_{2 i}^{Q}\right)$ between the pre-impact velocities projected onto the line of impulse. This in fact is precisely the condition of restitution stated in equation 3.12 (equation 3.6 for the standard model) and pictured on the right-hand side of figure 2. It is not part of the core model that $\left(\boldsymbol{v}_{1 f}^{Q}-\boldsymbol{v}_{2 f}^{Q}\right)$ should be parallel to the impulse; this is a strengthening of the basic condition of restitution (equations 2.5 and 3.6 as shown on the left-hand side of figure 2) to exclude any tangential component of relative motion, i.e. sliding of the contacting surfaces, and is required on the Smith-Neades model [19, eqn 25] for the computation of relative velocity, including closing speed.

\section{Discussion}

The illustrative case described above is a single example and there should be no temptation to generalise the magnitude of effect observed there. It can be foreseen that the consequences of shifting restitution from the middle of the crush zone to the residual crush surface will vary widely according to the impact details. The main point was to link the theory presented above to the literature through a concrete case and to show that a modest adjustment to a realistic dataset can produce a discernible effect.

The RICSAC trials and many subsequent crash tests have been used to validate and refine accident reconstruction models and techniques, however the purpose of separating the restitution point $\mathrm{Q}$ from the impulse point $\mathrm{P}$ was not to have a new parameter and degree of freedom for optimising computed results against experimental data. The primary motivation was an engineering assessment that the fidelity of the model would be deepened by this extension of its scope, making for a more realistic accident reconstruction. Furthermore the location of the point $\mathrm{Q}$ is fully determined by the crush profile and direction of impulse from $\mathrm{P}$ - the refined model as described offers no additional degree of freedom or element of discretion in its use. In contrast it makes good engineering and mathematical sense that impulse be located in the central region of the crush zone given that it is related to an average value of collision force over the whole duration of impact.

In the context of long-running, systematic research studies such as those sponsored by the Department for Transport in Great Britain previously mentioned [5-7], the 'null' result for the effect of point $\mathrm{Q}$ on velocity change might at first glance seem to diminish its significance. In fact the opposite is true. Firstly it deepens understanding of the robustness of the basic model by showing how delta- $\mathrm{V}$ is insensitive in a certain way to the location of vehicle engagement. Secondly it shows that expanding the core damage-based reconstruction beyond the traditional scope of the Crash3 algorithm to include closing speed (relative velocity) and road speed (absolute velocity) entails a close review of the underlying mathematical model. Thirdly and of most direct relevance to many stakeholders, thousands of accidents have been reconstructed in the research studies since 1983 and the results are embedded in injury risk curves, assessments of the effectiveness of safety technologies and the description of common accident scenarios for the development of test conditions, to name just a few applications. To know that these analyses, in so far as they as based on velocity change, are insulated against changes in the treatment of the restitution point, and to know that the databases will not be subject to a step change that breaks continuity with the past for this reason, is very significant.

In order to isolate the effect of separating impulse from restitution, it was considered important 
to adhere closely to a mainstream model while at the same time adopting a mathematical style amenable to the exploration of theoretical refinements. In fact equation 3.5, which provides the key to solving for change of velocity, may be transformed through the substitution of $I=m k^{2}$ where $k$ is the radius of gyration, $\boldsymbol{J}=m \Delta \boldsymbol{v}, \boldsymbol{R} \times \hat{\boldsymbol{J}}=\boldsymbol{h}$ and $\delta=1+\frac{h^{2}}{k^{2}}$ to obtain

$$
\begin{aligned}
\frac{(1+e)}{(1-e)} E & =\frac{1}{2}\left(\frac{1}{m_{1}}+\frac{\boldsymbol{h}_{1}^{2}}{m_{1} k_{1}^{2}}+\frac{1}{m_{2}}+\frac{\boldsymbol{h}_{2}^{2}}{m_{2} k_{2}^{2}}\right)(m \Delta \boldsymbol{v})^{2} \\
& =\frac{1}{2}\left(\frac{\delta_{1}}{m_{1}}+\frac{\delta_{2}}{m_{2}}\right)\left(m_{1} \Delta \boldsymbol{v}_{1}\right)^{2}
\end{aligned}
$$

or

$$
\left\|\Delta \boldsymbol{v}_{1}\right\|=\sqrt{\frac{2(1+e) E m_{2}}{(1-e) m_{1}\left(m_{1} \delta_{2}+m_{2} \delta_{1}\right)}}
$$

This corresponds in form to the main result in [22], a generalisation of 'the formula commonly used to calculate velocity change' to include restitution and confirms the correction subsequently noted regarding the index numbers of the delta terms [19]. The closeness of equation 4.1 to equation 3.5 is by no means apparent at first sight and the same is true of their derivations. While this may be of little consequence to specialists in the field, it is thought beneficial to have a diversity of mathematical styles on offer to new entrants seeking a mastery of the material.

Finally it may be noted that the location of restitution on the residual damage surface at its intersection with the line of action of impulse was not the only option. There is no obstacle to using maximum dynamic crush if this is preferred and can be determined. More difficult is moving point $\mathrm{Q}$ away from the impulse line. The equations that solve for an arbitrary point on the rigid body are considerably longer and more complex than those presented above. For current purposes the remarkable simplicity of restitution on the impulse line was judged to outweigh a more general result, especially with the intuitive plausibility from an engineering standpoint that engagement of the contacting surfaces should be well defined close to and in the direction of the net impact force.

\section{Conclusion}

In the standard mathematical model that underpins the inference of velocity change from vehicle damage, the point at which the colliding vehicles are assumed to engage is located in the same place as the impact force. A minimal modification of the theory to separate these points, with engagement on the residual crush surface and force in the central crush zone, revealed that the solutions for linear and angular velocity change are unaffected. This means that long-running in-depth accident studies can in principle adopt the modification without putting at threat historical calculations of velocity change and analyses for multiple stakeholders based on these results. More recent extensions of the core model to include relative velocity (closing speed) and absolute velocity (road speed) are however affected by separation of the restitution point. This finding was illustrated by reference to a two-vehicle crash test where altering the standard location of the point of restitution by up to twelve centimetres resulted in a change of up to four percent in the assessment of road speed.

\section{Acknowledgements}

The author expresses his gratitude to Alexandro Badea Romero for constructive advice on the structure, content and presentation of the manuscript as it evolved. 


\section{References}

[1] M. Alonso and E.J. Finn, Fundamental University Physics: Mechanics and Thermodynamics, Vol. 1, 2nd ed., Addison-Wesley, 1980.

[2] F. Böhm, Der nichtzentrale Stoß von zwei luftbereiften Fahrzeugen: Teil 1, Automobiltechnische Zeitschrift 70 (1968), pp. 385-9.

[3] R.M. Brach and R.M. Brach, A review of impact models for vehicle collision, technical paper 870048 , Society of Automotive Engineers, 1987.

[4] R.M. Brach and R.M. Brach, Vehicle Accident Analysis and Reconstruction Methods, SAE International, Warrendale, Pennsylvania, 2005.

[5] Department for Transport, Research database: On the Spot Accident Data Collection phase 1 (2004), URL http: //www.dft.gov.uk/rmd/project.asp?intProjectID=10801.

[6] Department for Transport, Research database: Co-operative Crash Injury Study (CCIS) phase 8 (2006), URL http://www.dft.gov.uk/rmd/project.asp?intProjectID=12088.

[7] Department for Transport, Research database: Road Accident In-Depth Studies (RAIDS) (2013), URL http://www.dft.gov.uk/rmd/project. asp?intProjectID=12962.

[8] G. Grime and I.S. Jones, Car collisions - the movement of cars and their occupants in accidents, Proc IMechE 184 Part 2A (1969-70), pp. 87-125.

[9] W. Hugemann, Kollisionsmechanik, in Unfallrekonstruktion, W. Hugemann, ed., Vol. 1, chap. 2.2, Verlag autorenteam, Münster, 2007, p. 227.

[10] H. Ishikawa, Impact model for accident reconstruction-normal and tangential restitution coefficients, technical paper 930654, Society of Automotive Engineers, 1993.

[11] H. Ishikawa, Impact center and restitution coefficients for accident reconstruction, technical paper 940564, Society of Automotive Engineers, 1994.

[12] I.S. Jones and A.S. Baum, Research input for computer simulation of automobile collisions (vol. IV), Tech. Rep. DOT HS 805 040, Department of Transportation, 1978.

[13] E. Marquard, Zur Mechanik des Zusammenstoßes von Fahrzeugen, Automobiltechnische Zeitschrift 64 (1962), pp. 141-8.

[14] R.R. McHenry, A comparison of results obtained with different analytical techniques for reconstruction of highway accidents, Tech. Rep. 750893, Society of Automotive Engineers, 1975.

[15] National Highway Traffic Safety Administration, Crash 3 technical manual (1986).

[16] National Highway Traffic Safety Administration, National Automotive Sampling SystemCrashworthiness Data System: 2013 coding and editing manual (2014), DOT HS 812067.

[17] J. Neades, Developments in road vehicle crush analysis for forensic collision investigation, Ph.D. thesis, Faculty of Technology, De Montfort University, 2011.

[18] J. Neades, Equivalence of impact-phase models in two-vehicle planar collisions, Proc IMechE Part D: Journal of Automobile Engineering 227 (2013), pp. 1325-1336.

[19] J. Neades and R. Smith, The determination of vehicle speeds from delta- $V$ in two vehicle planar collisions, Proc IMechE Part D: Journal of Automobile Engineering 225 (2011), pp. 43-53.

[20] E.J. Routh, The Elementary Part of a Treatise on the Dynamics of a System of Rigid Bodies, Macmillan, 1882.

[21] A. Slibar, Die mechanischen Grundsätze des Stoßvorgangs freier and geführter Körper und ihre Anwendung auf den Stoßvorgang von Fahrzeugen, Archiv für Unfallforschung 2 (1966), pp. 31-53.

[22] R. Smith, The formula commonly used to calculate velocity change in vehicle collisions, Proc IMechE Part D: Journal of Automobile Engineering 212 (1998), pp. 73-8. 